\title{
ASTRONOMICAL APPLICATIONS OF CCDs IN HUNGARY - THE FIRST STEPS AND FUTURE PLANS
}

\author{
G. Szécsényi-Nagy \\ Eötvös Loránd University
}

\section{INTRODUCTION}

The first century of non-visual astronomical image detection in Hungary was devoted exclusively to photography. The most famous Hungarian astronomers and astrophysicists of the last century were the pioneers of astrophotography too. The very first exhaustive description of photography as a scientific method was the one by Konkoly-Thege about astronomical photography in 1887. In his book one can find some interesting images printed of the plates taken by his friend and colleague, the enthusiastic astrophotographer J. Gothard, who was the first to demonstrate that the well-known bright planetary in Lyra (M 57 or the Ring Nebula) contains an extremely faint central star. His discovery, which opened new fields for astrophysics, was contested for a long time because his foreign contemporaries were unable to repeat this feat of arms.

Astrophotography remained, since then, the number one method in collecting observational evidence for scientific studies. World-known results of the Hungarian astronomical programs range from discoveries of minor planets, comets and flare stars to the first observations of novae and extragalactic supernovae. Photospheric and chromospheric features of the Sun were also amongst the targets of Hungarian sky-photographers during past decades.

\section{THE ADVENT OF ELECTRONIC IMAGING}

The first step into the field of electronic imaging was done during the late 1980s. The capabilities of photographic and photoelectric image detecting techniques were compared and as a result of it the introduction of CCD technology was suggested (especially to spectroscopic investigation and H-alpha photometry of dK and dM stars (Szécsényi-Nagy 1990 and 1994). Political changes made possible then the purchase of state-of-the art cooled CCD cameras, at least in principle. The budget of astronomical institutes remained the last restriction in planning this step of modernization and unfortunately this limit has been fixed at a very low level. Consequently it is no wonder that the first cameras were simple cheap commercial products. These systems have TV-image resolution and very low dynamic range (the A/D conversion offered is normally eight bits). As has been found at the Baja Observatory e.g., the uncooled EDC1000HR CCD camera fits the needs of astronomical demonstration and education. Possible targets are the Moon, the Sun and the planets or a bright comet (Hegedüs 1994). The University of Szeged (JATE) bought a chilled ST-4 CCD Star Tracker/Imaging Camera. This device is so light that it could be mounted on a $63 / 840 \mathrm{~mm}$ visual refractor (Telemator made by Carl Zeiss Jena). The ST-4 contains a very small chip (its active area is only $2.5 \mathrm{~mm} \times 2.5$ 
$\mathrm{mm}$ ) and its short focal length is definitely advantageous when the program needs an extended field of view. The power of the system has been tested on globular clusters, planetary nebulae and galaxies. The photometry of the SN in M 81 and that of a faint mira (DQ Vul) has been performed by this device also (Szatmary 1994). Their next step is to purchase a more sophisticated version of that product an ST-6. The JATE team intends to use this new system for the photometry of pulsating variables.

Our institute, the Department of Astronomy of the Eötvös University, received a thermoelectrically cooled eight-bit CCD camera too. Unfortunately the chip of the device had been constructed for TV cameras. The greatest problem is that the CCD has an interlaced structure and their pixels are not square. Furthermore its spectral sensitivity is negligible in the violet and UV range relative to the dark noise of the device. Otherwise it is a reliable system. For comparison we were able to record an image of the M 57 with an exposure which is shorter than $1 \%$ of the photographic exposure that Gothard had to apply a century ago. Another drawback of the camera is the lack of any optical filters. This also limits the scientific use of the system. Our present efforts are aimed at ordering a more sophisticated camera with at least 12 bit A/D conversion and a larger chip (1 Megapixel), better cooling and a computer controlled filter wheel with a set of UBVRI, $\mathrm{H}$-alpha and H-beta filters.

\section{ACKNOWLEDGEMENT}

The author acknowledges the partial support of this research by the NSRF (grants: OTKA-T 7595 and OTKA-U 15909).

\section{REFERENCES}

Hegedüs, T. 1994, private communication

Szatmary, K. 1994, private communication

Szécsényi-Nagy, G. 1990 Star Clusters and Associations, Balazs, B., and Szécsényi-Nagy, G., eds., ELTE, Budapest, p. 55

Szécsényi-Nagy, G. 1994 Astronomy from Wide-Field Imaging, MacGillivray, H. T. et al., eds. Kluwer Academic Pub., Dordrecht, p. 61 